\title{
Medical and Legal Implications of Neuroelectric Research into Human Sexuality
}

\author{
Barry R. Komisaruk ${ }^{1}$ D
}

Received: 13 June 2020 / Revised: 23 June 2020 / Accepted: 25 June 2020 / Published online: 10 July 2020

(c) Springer Science+Business Media, LLC, part of Springer Nature 2020

The Target Article by Ziogas, Habermeyer, Santtila, Poeppl, and Mokros (2020) is a comprehensive review of "neuroelectrical" correlates, measured in brain, of responses to genital, visual, and other erotic stimulation. In their text and extensive comprehensive summary tables, Ziogas et al. carefully, and in great detail, review the findings of more than 250 studies in the literature. At the outset, they usefully characterize and describe the rationale for the variety of neuroelectrical recording methods that have been used, including quantitative analyses of EEG frequencies and amplitude, evoked responses to genital and erotic stimuli, event-related potentials, and several different methods of direct brain stimulation. Ziogas et al. review neuroelectrical studies of mapping genital representation in the brain, gender differences, correlates of sexual arousal and orgasm, epileptic seizures that induce, and that are induced by, orgasms, sexual behavior during sleepwalking, disturbances in sexual behavior, and "abnormal sexual preferences."

With regard to orgasm, Ziogas et al. (2020) review studies that showed changes in frequency and amplitude of delta, theta, alpha, and gamma EEG, activity of cortical regions (e.g., prefrontal, temporal, and parietal), and left-right brain asymmetrical activity. However, the functional significance of these specific changes to the perceptual and bodily physiological changes in orgasm is not addressed.

In focusing on neuroelectrical correlates of sexual responses and their unique value in temporal, rather than spatial, resolution of responses, Ziogas et al. (2020) ignore functional magnetic resonance imaging (fMRI) and positron emission tomography (PET) studies of sexual arousal and orgasm, which report much higher spatial resolution of

The original article of the commentary is https://doi.org/10.1007/ s10508-019-01547-3.

Barry R. Komisaruk

brk@psychology.rutgers.edu

1 Department of Psychology, Rutgers University, Newark, NJ 07102, USA regional brain responses. This suggests that a primary intent of their review was to explore the potential value of the neuroelectrical methodologies for possible diagnostic purposes. While these methodologies have long been used clinically to ascertain epileptic foci, Ziogas et al. raise the important question of whether the methodologies are valid for medicolegal application. What is the legal responsibility of an individual who commits rape or other sexual violations while sleepwalking, a condition confirmed by his EEG evidence? What are the legal implications for an individual who shows EEG activity characteristic of sexual arousal, when he is shown videos of children but not adults?

While Ziogas et al. (2020) do not explicitly advocate the use of these methodologies for such applications, they devote significant text to evidence related to neuroelectrical correlates of sexual behavior considered to be pathological. This raises, but does not answer, the implied question of whether such evidence could or should be used for medicolegal purposes. One would hope that non-neuroscientists would not be led to believe that, because sophisticated technology is used to record brain activity, the neuroelectrical methods can provide evidence leading to valid conclusions on which to base important medical and legal decisions. It would have been helpful had Ziogas et al., presumably aware of the potential of this technology for medicolegal abuse, provided such caveats in their exhaustive review.

\section{Reference}

Ziogas, A., Habermeyer, E., Santtila, P., Poeppl, T. B., \& Mokros, A. (2020). Neuroelectric correlates of human sexuality: A review and meta-analysis. Archives of Sexual Behavior. https://doi. org/10.1007/s10508-019-01547-3.

Publisher's Note Springer Nature remains neutral with regard to jurisdictional claims in published maps and institutional affiliations. 